\title{
Projective planes with a transitive automorphism group
}

go back

full screen

close

quit

\author{
Alan R. Camina
}

\begin{abstract}
In this note we prove two theorems which contribute towards the classification of line-transitive designs. A special class of such designs are the projective planes and it is this problem which the paper addresses. There two main results:-

Theorem A: Let $G$ act line-transitively on a projective plane $\mathcal{P}$ and let $M$ be a minimal normal subgroup of $G$. Then $M$ is either abelian or simple or the order of the plane is $3,9,16$ or 25 .

Theorem B: Let $G$ be a classical simple group which acts line-transitively on a projective plane. Then the rank of $G$ is bounded.
\end{abstract}

Keywords : projective planes, simple groups

MSC 2000: 51A35 (primary), 20B25 (secondary)

\section{Introduction}

This paper is part of an ongoing programme to classify line-transitive automorphism groups of finite linear spaces. A special class of such spaces are the projective planes. This problem ought to be straightforward. In a classical paper [14] A. Wagner has shown that if there are perspectivities then the plane is Desarguesian. It is then easy to see that the group of automorphisms, say $G$, is a subgroup of $\operatorname{PGL}(3, q)$ containing $\operatorname{PSL}(3, q)$ for some prime-power $q$.

Now let $G$ be a line-transitive automorphism group of a projective plane of order $n$. Then an involution in $G$ is either a perspectivity or a Baer involution and will fix a subplane of order $\sqrt{n}$. It would appear that this is a strong condition but seems to give less information than one might expect. In fact Kantor in [9] was unable to use this fact when considering the case when $G$ is primitive. 



\section{Minimal Normal Subgroups}

This section is devoted to the proof of Theorem 1.1. We note that by [1, Theorem 2] $G$ contains at most one non-abelian normal subgroup. We have to show that if there is such a non-abelian normal subgroup then it is simple.

So assume that $M$ is neither abelian nor simple. Then we can write $M=$ $M_{1} \times M_{2} \times \cdots \times M_{d}$ where $d>1$ and each $M_{i}$ is isomorphic to some simple group. Let the projective plane have order $n$. We know from [1, Theorem 1] that normal subgroups of $G$ act faithfully on each orbit. Let $N$ be a normal subgroup of $M$. The set of fixed points of $N$ is the union of orbits of $M$ and so $N$ would be in the kernel of the action of some orbit of $M$, contradicting the assertion that $M$ is faithful. Further since each $M_{i}$ is simple, if it were to act unfaithfully on an orbit it would have fixed points. This contradicts the assertion above. Hence we may assume that each $M_{i}$ acts without fixed points and faithfully on each of its orbits.

Let $T$ be a Sylow 2-subgroup of $M$. Since $\mathcal{P}$ has an odd number of points we see that there is a point $P$ so that $T$ fixes $P$. Thus $\left(M_{i}\right)_{P} \neq 1$ for $1 \leq i \leq d$. Since $G$ acts transitively on $\mathcal{P}$ this must be true for all points $P$.

Choose $P$ so that $\left(M_{1}\right)_{P}$ has maximal order. We observe that $\left[M_{2},\left(M_{1}\right)_{P}\right]=1$ so $P M_{2}$ consists of points fixed by $\left(M_{1}\right)_{P}$. Since $M_{j}$ is faithful on all its orbits, on points or lines, we see that $P M_{2}$ contains at least 5 points as $M_{2}$ is simple non-abelian. Thus $\left(M_{1}\right)_{P}$ fixes at least one line through $P$. The fixed set is either a subplane or there exists a pair $(P, l)$ where $l$ is a line and every other point fixed by $\left(M_{1}\right)_{P}$ will be on $l$ and every other fixed line will be incident with $P,[4,3.1 .2]$. But as $M_{2}$ does not fix any point or line we see that $\left(M_{1}\right)_{P}$ fixes a subplane whose order is at most $\sqrt{n}$, by Lemma 1.3

We now claim that for any line $m$ incident with $P$ there is a $j$ so that $\left(M_{j}\right)_{P}$ fixes $m$. Choose a line $m$ incident with $P$. If $\left(M_{1}\right)_{P}$ fixes $m$ there is nothing to prove. Let $m_{1}$ be a line incident with $P$ fixed by $\left(M_{1}\right)_{P}$, there is one by the above argument. But $G$ is transitive on lines so there is $g \in G$ with $m_{1} . g=m$. Then $Q=P g$ is incident with $m$ and $\left(\left(M_{1}\right)_{P}\right)^{g}$ fixes $m$. But there exists $j$ so that $\left(\left(M_{1}\right)_{P}\right)^{g}=\left(M_{j}\right)_{Q}$ since $g$ permutes the factors $M_{i}$. Let $i \neq j$. Then $\left(M_{i}\right)_{P}$ commutes with $\left(M_{j}\right)_{Q}$ and so fixes the set of lines fixed by $\left(M_{j}\right)_{Q}$. If $\left(M_{i}\right)_{P}$ fixes $m$ then we have proved our claim. If not we see that $\left(M_{j}\right)_{Q}$ fixes at least two lines through $P$ and so fixes $P$. However $\left(\left(M_{1}\right)_{P}\right)^{g}=\left(M_{j}\right)_{Q}$ so by the maximality of $\left(M_{1}\right)_{P}$ we have that $\left(M_{j}\right)_{Q}=\left(M_{j}\right)_{P}$ and the claim is proved.

We now count that each $\left(M_{j}\right)_{P}$ fixes at most $\sqrt{n}+1$ lines and so $d(\sqrt{n}+1) \geq$ $n+1$. So $d \geq \sqrt{n}$. Since $M$ has an abelian subgroup of order at least $5^{d}$ it follows from Lemma 1.5 that $n^{2}+n+1 \geq 5^{d} \geq 5^{\sqrt{n}}$. This has no solutions. 


\section{Classical Simple Groups}

Combining Lemmas 1.4 and 1.5 we get the following useful lemma.

Lemma 3.1. Let $G$ act line-transitively on a projective plane of order $n$. Assume that $G$ contains an involution $s$ with $w$ conjugates in $G$ and an abelian subgroup A of order $a$. Then $a \leq 4 w^{2}$.

Proof. Let $z$ be the number of conjugates of $s$ in $G_{P}$, where $G_{P}$ is the stabiliser of the point $P$. By counting we have that, using Lemma 1.4,

$$
\left(n^{2}+n+1\right) z=(n+\sqrt{n}+1) w .
$$

So we have the inequality $n-\sqrt{n}+1 \leq w$. This gives $\sqrt{n}<\sqrt{w}+1$. So $n<3 w / 2$ and $n^{2}+n+1<4 w^{2}$.

We now use Lemma 1.5 to see that $a \leq n^{2}+n+1$ and so the result follows.

Thus to prove Theorem 1.2 we need to find large abelian subgroups and involutions with a small number of conjugates.

In the next lemma we list sizes of known abelian subgroups for the classical groups.

Lemma 3.2. The following lists orders of some abelian subgroups for the relevant groups. So in each case we have a lower bound for $a$.

1. $\operatorname{PSL}(2 d+1, q): a \geq q^{d(d+1)}$.

2. $\operatorname{PSL}(2 d, q): a \geq q^{d^{2}}$.

3. $\operatorname{PSp}(2 d, q): a \geq q^{(d(d+1)) / 2}$.

4. $P S U(2 d+1, q): a \geq q^{d(d+1)}$.

5. $\operatorname{PSU}(2 d, q): a \geq q^{d(d-1)}$.

6. $\Omega(2 d+1, q): a \geq q^{d(d-1) / 2}$.

7. $\Omega^{+}(2 d, q): a \geq q^{d(d-1) / 2}$.

8. $\Omega^{-}(2 d, q): a \geq q^{(d-1)(d-2) / 2}$

Proof. For the estimates we need it is not hard to get the values above by considering certain matrix subgroups and using the forms given in Chapter 2 of [10]. 


\title{
References
}

[1] A. R. Camina and C. E. Praeger, Line-transitive automorphism groups of linear spaces. Bull. London Math. Soc. 25 (1993), 309-315.

[2] A. R. Camina and F. Spiezia, Sporadic groups and automorphisms of linear spaces. J. Combin. Des. 8 (2000), 353-362.

[3] A. R. Camina, P. M. Neuman and C. E. Praeger, Alternating groups acting on linear spaces. Proc. London Math Soc 87 (2003), 29-53.

[4] P. Dembowski, Finite Geometries, Springer-Verlag, Berlin, 1997. Reprint of the 1968 original.

[5] A. Gonçalves and C. Y. Ho, Alternating groups as collineation groups, $J$. Algebra 225 (2000), 581-601.

[6] M. Hall, Jr, The Theory of Groups. The Macmillan Co., New York, N.Y., 1959.

[7] C. Hering, On the structure of finite collineation groups of projective planes, Abh. Math. Sem. Univ. Hamburg 49 (1979), 155-182.

[8] D. R. Hughes and F. C. Piper, Projective Planes, Graduate Texts in Mathematics, Vol. 6, Springer-Verlag, New York, 1973.

[9] W. M. Kantor, Primitive permutation groups of odd degree, and an application to finite projective planes, J. Algebra 106 (1987), 15-45.

[10] P. Kleidman and M. Liebeck, The Subgroup Structure of the Finite Classical Groups, Cambridge University Press, Cambridge, 1990.

[11] A. R. Prince, Strongly irreducible collineation groups and the Hall-Janko group, Period. Math. Hungar. 23 (1991), 99-104.

[12] A. Reifart and G. Stroth, On finite simple groups containing perspectivities, Geom. Dedicata 13 (1982), 7-46.

[13] G. Stroth, On Chevalley groups acting on projective planes, J. Algebra 77 (1982), 360-381.

[14] A. Wagner, On projective planes transitive on quadrangles, J. London Math. Soc. 33 (1958), 25-33.

\author{
Alan R. Camina \\ School of Mathematics, University of EAst ANglia, Norwich NR4 7TJ \\ e-mail: a.camina@uea.ac.uk
}

